УДК 62-97/98

\title{
МЕТОД И СРЕДСТВА ИСПЫТАНИЙ КАВИТАЦИОННОЙ ЭРОЗИИ В АНОМАЛЬНЫХ УСЛОВИЯХ
}

\author{
Хмелев Владимир Николаевич 1 , \\ vnh@bti.secna.ru
}

\author{
Барсуков Роман Владиславович 1 , \\ roman@bti.secna.ru
}

Голых Роман Николаевич', grn@bti.secna.ru

\author{
Абраменко Денис Сергеевич1, \\ ades@bti.secna.ru
}

Генне Дмитрий Владимирович1', gdv@bti.secna.ru

\author{
Тертишников Павел Павлович 1 , \\ tertishnikov.pp@bti.secna.ru \\ 1 Алтайский государственный технический университет им. И.И. Ползунова,
Россия, 659305, г. Бийск, ул. Трофимова, 27.
}

\begin{abstract}
Актуальность исследования обусловлена необходимостью изучения стойкости металлов и их покрытий к кавитационной эрозии в условиях аномально высоких температур, давлений, а также в химически агрессивных средах в виде кислот, щелочей или агрессивных газов (типа озона).

Цель: создание специализированного оборудования для контроля и выявления режимов и зависимостей кавитационного разрушения различных металлов и их покрытий при аномально высоких температурах и избыточном давлении, а также методологии определения (контроля) стойкости металлов и их покрытий в кавитационных полях.

Объекты: процесс испытания кавитационной эрозии в аномальных условиях с помощью специально разработанного ультразвукового оборудования для данной задачи.

Метод: экспериментальный метод исследования кавитационной эрозии в аномальных условиях, который является развитием существующего метода, регламентированного стандартом ASTM G32-10 «Стандартный метод испытаний кавитационной эрозии».

Результаты. Для создания, исследования и применения специальных материалов и покрытий, способных обеспечить длительную надежную эксплуатацию оборудования предложен и разработан новый способ контроля эрозионной стойкости металлов и защитных покрытий при кавитационном воздействии в аномальных, по давлению и температуре, эксплуатационных условиях, расширяющий возможности стандарта ASTM G32-10 «Стандартный метод испытаний кавитационной эрозии». Практическая реализация испытаний кавитационной эрозии металлов и защитных покрытий в аномальных условиях обеспечит создание и применение новых материалов для использования в различных отраслях деятельности человека, промышленности.
\end{abstract}

\section{Ключевые слова:}

Ультразвуковое воздействие, акустическое поле, эрозия, экстремальные условия, частота, нормальные условия, амплитуда.

\section{Введение}

Стойкость металлов и их защитных покрытий при воздействии концентрированных потоков вещества и энергии является залогом долгой и надежной работы деталей механизмов и машин, используемых в самых различных отраслях промышленности.

Одним из возможных механизмов разрушения металлов и покрытий является кавитация, которая представляет собой процесс образования и последующего схлопывания парогазовых пузырьков в жидкости. Это явление встречается повсеместно, поскольку гидродинамическая кавитация возникает в результате локального понижения давления в жидкости, за счет движущихся в ней элементов различных механизмов (насосов, например) или при обтекании жидкостью неподвижных элементов, а акустическая кавитация возникает в жидких средах при распространении в них акустических колебаний большой интенсивности.

Кавитационный процесс на поверхностях изделий (покрытий) сопровождается рядом вторичных явлений, к числу которых относятся: ударные волны, кумулятивные струи, локальное повышение температуры, электрические разряды, выделяющиеся газы, которые могут быть агрессивными или способствовать окислительным процессам. Все эти явления негативно сказываются на состоянии поверхностей элементов, деталей и машин, непосредственно находящихся или работающих в жидкой среде, приводят к быстрому износу реакторов, теплообменников, трубопроводов, насосов и других элементов, снижают производительность оборудования, сокращают сроки его эксплуатации. 
Ситуация существенно осложняется тем, что жидкие среды могут иметь аномально высокую температуру, находиться под большим избыточным давлением, быть химически агрессивными из-за наличия в них химических реагентов в виде кислот, щелочей или агрессивных газов (типа озона).

На практике реализуется два основных подхода к решению проблемы кавитационного износа:

- применение специальных материалов и покрытий;

- создание условий, обеспечивающих снижение кавитационного воздействия за счет усложнения конструкций, снижения скоростей потоков и т.п.

Второй подход не всегда применим, поскольку накладывает определенные ограничения на режимы работы оборудования и не позволяет полностью исключить влияние кавитационных явлений на элементы и узлы гидравлических систем.

Поэтому основные усилия создателей нового специализированного оборудования для специальных отраслей промышленности (атомная, химическая, металлургическая) направлены на поиск и применение таких материалов и покрытий, которые способны обеспечить длительную надежную эксплуатацию оборудования, в том числе в условиях кавитационного воздействия.

Выбор и поиск таких материалов нельзя обеспечить из-за отсутствия экспериментальных результатов по кавитационной эрозии различных материалов и покрытий при высоких температурах и давлениях. К сожалению, в настоящее время такие данные невозможно получить из-за отсутствия оборудования, способного создать кавитационные воздействия при высоких давлениях, температуре до 1000 градусов Цельсия и в различных по свойствам жидких средах (в том числе - агрессивных).

В связи с этим возникает необходимость создания специализированного оборудования для контроля и выявления режимов и зависимостей кавитационного разрушения различных металлов и их покрытий при аномально высоких температурах и избыточном давлении, а также создания методологии определения (контроля) стойкости металлов и их покрытий в кавитационных полях. Существует масса исследований, посвященных проблеме кавитационной стойкости различных материалов [1-15].

В настоящее время для определения степени кавитационной эрозии материалов и их покрытий применяется стандарт ASTM G32-10 «Стандартный метод испытаний кавитационной эрозии» [16], который регламентирует проведение исследований и определяет требования к применяемому оборудованию.

Для реализации стандартного метода испытаний кавитационной эрозии при воздействии кавитации в воде при нормальных условиях (температура и давление) предложено и разработано специализированное оборудование [17, 18].

К сожалению, использовать его для практических исследований, в соответствии с указанными требованиями, невозможно без решения следующих основных задач:
- обеспечения возможности проведения исследований в высокотемпературных и агрессивных средах с применением специальных рабочих инструментов и в специальных технологических объемах;

- создания и применения средств контроля и управления амплитудой колебаний инструмента в различных средах и условиях для обеспечения равенства создаваемого ультразвукового воздействия;

- обеспечения равного по эффективности кавитационного процесса (степени развитости кавитации) в зазоре между излучающим инструментом и исследуемым образцом в различных средах и при различных условиях за счет управления кавитационным процессом.

Поскольку без решения перечисленных задач реализация контроля кавитационной прочности материалов в аномальных условиях невозможна, далее представлены результаты исследований в этом направлении.

\section{Ультразвуковой аппарат для работы} в аномальных условиях

Для реализации кавитационного процесса при высоких температурах (до 1000 градусов) и давлениях в различных жидких средах (вода или агрессивные жидкости, масла, расплавы металлов и др.) крайне важно использовать специально разработанную и созданную для ультразвукового воздействия пьезоэлектрическую колебательную систему, её конструкция представлена на рис. 1.

Предложенная и разработанная в рамках данной научной проблемы конструкция УЗ излучателя [19], состоящая из пьезоэлектрического преобразователя, который осуществляет преобразование электрических колебаний в механические - 1 , концентратора, который усиливает механические колебания - 2, излучателя ультразвуковых колебаний - рабочего инструмента - 3 из ниобия, тантала или их сплавов длиной, не менее половины длины волны продольных колебаний ультразвука на частоте его возбуждения.

На концентраторе, в месте минимума механических колебаний, выполнен крепежный поясок - 4, симметрично оси концентратора и излучателя размещена охлаждающая камера - 5, выполненная в виде полого цилиндра с патрубками ввода -6 и вывода -7 охлаждающей жидкости. Одна из торцевых поверхностей -8 полого цилиндра охлаждающей камеры герметично закреплена на пояске концентратора - 4 . Вторая торцевая поверхность - 9 охлаждающей камеры - 5 имеет центральное отверстие и герметично закреплена при помощи эластичного уплотнения - 10 на поверхности излучателя, в месте, расположенном на расстоянии, равном четверти длины волны колебаний в излучателе от места соединения - 11 излучателя с концентратором.

Конструктивной особенностью аппарата является выполнение рабочего инструмента - 3 из ниобия, тантала и их сплавов, характеризующихся высокой химической стойкостью и температурой плавления, значительно превышающей (не менее 2 раз) темпера- 
туры плавления легких сплавов. Это позволяет решить проблему непосредственного ультразвукового воздействия на жидкие среды с температурой до 1000 градусов.

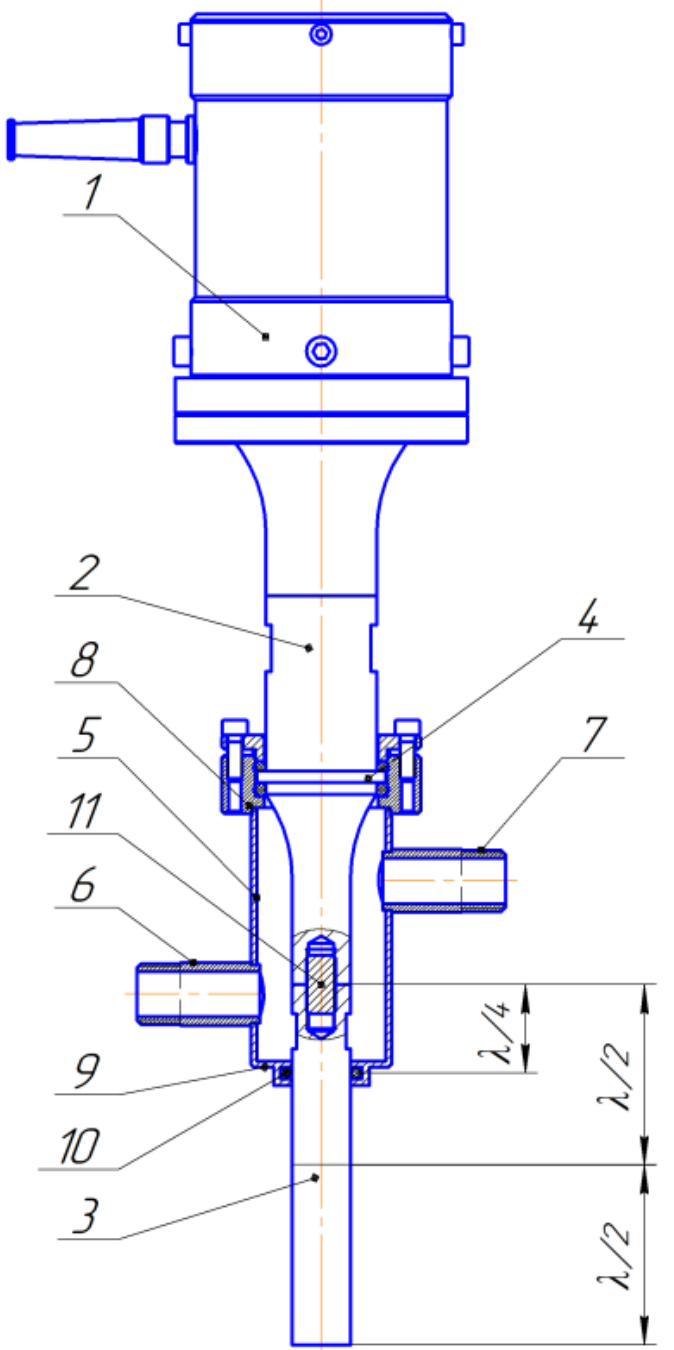

Pис. 1. Специально разработанная и созданная пьезоэлектрическая колебательная система ультразвукового оборудования для образования кавитаиионного процесса при различных аномальных условиях

Fig. 1. Specially designed and created piezoelectric oscillatory system of ultrasonic equipment for formation of cavitation process under various anomalous conditions

Предлагаемый аппарат обеспечивает длительную обработку агрессивных сред при высокой температуре без снижения эффективности ультразвукового воздействия.

При таком конструктивном исполнении исключена возможность нарушения акустического контакта между рабочим инструментом и концентратором при высокой температуре обрабатываемых сред, обеспечена стабилизация параметров преобразователя, концентратора и излучателя. При этом выполнение рабочего инструмента - излучателя - из высокотемпературного материала и резонансной длины, соответ- ствующей одной или двум длинам полуволн в материале инструмента, обеспечивает возможность размещения части инструмента и соединительной поверхности с концентратором внутри охлаждающей камеры, в которой протекает охлаждающая жидкость (например, водопроводная вода с температурой не выше 35 градусов). Это обеспечивает охлаждение концентратора, части рабочего инструмента, исключает нагрев преобразователя, что дает возможность использовать пьезоэлектрический преобразователь, имеющий больший КПД, в сравнении с преобразователем магнитострикционного типа.

Вторая торцевая поверхность охлаждающей камеры имеет центральное отверстие и герметично закреплена на поверхности излучателя в месте, расположенном на расстоянии, равном четверти длины волны колебаний в излучателе от места соединения излучателя с концентратором. Такое крепление исключает влияние охлаждающей камеры на резонансные свойства излучателя и всей системы и позволяет, при необходимости, легко заменять излучатель.

Подаваемые от электронного генератора ультразвуковой частоты электрические колебания преобразуются пьезоэлектрическим преобразователем - 1 в упругие колебания ультразвуковой частоты, усиливаются до амплитуды не менее 10 мкм. Затем упругие колебания усиливаются концентратором - 2 механических колебаний, имеющим соотношение диаметров 4:1, до величины не менее 50 мкм и передаются в рабочий инструмент - 3 резонансной длины. Колебания торцевой свободной поверхности излучателя, частично погружаемого в любые среды, с амплитудой не менее 50 мкм, обеспечивают интенсивность ультразвукового воздействия не менее $30 \mathrm{BT} / \mathrm{cm}^{2}$. Такой интенсивности воздействия достаточно для формирования кавитационного процесса в режиме развитой кавитации практически в любых жидких средах и при избыточных давления до 10 атм [20].

Проведенные испытания ультразвукового аппарата показали, что нарушения акустического контакта в процессе многодневной эксплуатации не происходит, параметры колебательной системы в процессе обработки любых сред (даже расплавов) стабилизируются, т. е. обеспечивается длительное воздействие без изменения параметров УЗ колебаний. Частичное охлаждение рабочего инструмента - излучателя - позволило увеличить срок его эксплуатации в средах при температуре 750 градусов не менее чем в 10-15 раз.

Для проведения исследований по кавитационной стойкости различных материалов и покрытий используется специальный герметичный технологический объем, в котором могут создаваться требуемые аномальные температуры и давления.

Стандартный метод основан на контроле изменения массы рабочего инструмента. Изготовление и применение инструментов из низкотемпературных, химически не стойких металлов, керамик, полимеров и т. п. не представляется возможным, поэтому необходимо применять альтернативный подход, при котором колеблющийся рабочий инструмент размещается в непосредственной близости от образца с исследуе- 
мым материалом или покрытием. При этом разрушение материалов или покрытий осуществляется за счет формирования кавитационного процесса в зазоре между торцевой поверхностью излучателя и поверхностью исследуемого образца.

Поскольку специальный герметичный технологический объем и создаваемые в нем условия исследований не позволяют осуществлять визуальное наблюдение или использовать внутри датчики контроля параметров ультразвукового воздействия, возникает необходимость создания и применения средств контроля и управления амплитудой колебаний рабочего инструмента в различных средах и условиях для обеспечения контроля и управления кавитационным процессом.

\section{Управление амплитудой колебаний инструмента в различных средах}

Во время акустического воздействия на всевозможные технологические среды при разных температурах и давлениях происходит изменение амплитуды механических колебаний ультразвуковых колебательных систем (УЗКС). Это связано с изменением волнового сопротивления зоны ультразвукового воздействия и резонансных свойств самой системы. Это обусловлено не только различиями в свойствах сред, нагревом работающей в исследуемом объеме части инструмента, но и возникновением непосредственно перед колебательной поверхностью области, состоящей из парогазовых кавитационных пузырьков.

Изменение резонансных свойств УЗ излучателя приводит к резкому снижению амплитуды ультразвуковых колебаний излучающей поверхности, что в конечном итоге приводит к снижению эффективности ультразвукового воздействия. В связи с этим необходимо контролировать и корректировать амплитуду УЗ воздействия, поддерживая ее на определенном уровне (например, равной 50 мкм, в соответствии с требованиями стандартного метода).

Таким образом, при проведении исследований кавитационной эрозии материалов, для обеспечения равных по амплитуде воздействий в различных средах и при различных условиях, необходимо управлять процессом ультразвукового воздействия.

Управление осуществляется путем изменения напряжения питания пьезопреобразователя для достижения необходимого уровня амплитуды колебаний рабочего инструмента.

Анализ современных методов косвенного контроля амплитуды колебаний рабочего инструмента ультразвуковой колебательной системы позволил выявить принципиальную возможность ее контроля по электрическому току, протекающему по динамической (виртуальной) ветви схемы замещения УЗ излучателя.

В процессе ультразвукового воздействия критерием настройки УЗ генератора на резонансную частоту У3 излучателя является равенство нулю сдвига фаз между напряжением на УЗ излучателе и током $I$, протекающим в динамической (виртуальной) ветви УЗ излучателя, образованной элементами $L_{0}, C_{0}, R_{0}, L_{\mathrm{H}}$, $C_{\mathrm{H}}, R_{\mathrm{H}}$ (рис. 2).

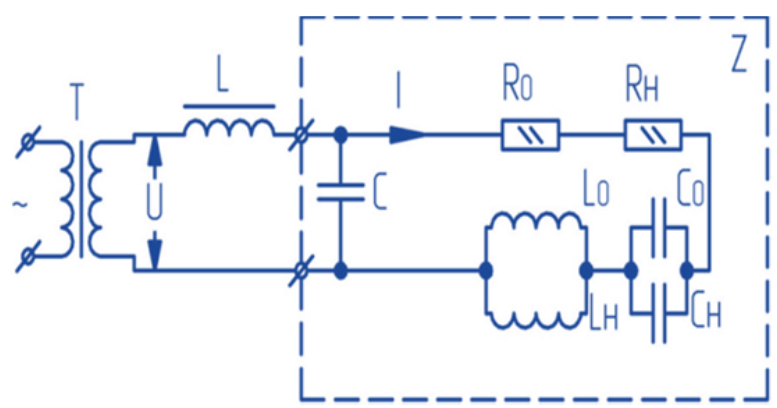

Рис. 2. Физическая эквивалентная схема замещения УЗ излучателя

Fig. 2. Physical equivalent circuit of ultrasonic emitter substitution

На рис. 2 иллюстрируется физическая эквивалентная схема замещения УЗ излучателя. Представленная схема замещения справедлива в окрестностях резонансной частоты реального УЗ излучателя. Как видно из схемы, она имеет комплексное сопротивление $Z$. Реактивные элементы $L_{0}, C_{0}, L_{\mathrm{H}}, C_{\mathrm{H}}$ являются электрическими эквивалентами массы УЗ излучателя, упругости материала УЗ излучателя, массы присоединенной к УЗ излучателю обрабатываемой жидкой среды и упругости обрабатываемой жидкой фазы соответственно. Активные элементы схемы $R_{0}$, $R_{\text {н }}$ обусловлены потерями в материале УЗ излучателя и активным сопротивлением акустической нагрузки соответственно, причем значение элемента $R_{\mathrm{H}}$ определяется не только физическими свойствами озвучиваемых жидких сред, но и степенью развития в них кавитационных явлений, возникновение которых сопровождается разрыхлением сплошной жидкой среды парогазовыми пузырьками. Влияние кавитационных явлений на значение элемента $R_{\mathrm{H}}$ имеет большую практическую значимость, поскольку появляется возможность косвенного контроля эффективности кавитационного процесса. Элемент $C$ является реальным и определяется статической емкостью преобразователя. Элементы $L$ и $T$ являются согласующей трансформаторно-дроссельной схемой, при помощи которой УЗ излучатель подключается к электронному генератору.

Для контроля амплитуды колебаний УЗКС, как было отмечено ранее, используется контроль тока динамической ветви схемы замещения УЗКС. Для выделения тока динамической ветви, для более качественного управления амплитудой УЗ излучателя и более точной работы системы ФАПЧ электронного генератора, как правило, применяются либо дифференциальные трансформаторы, либо дифференциальные схемы на операционных усилителях.

Таким образом, косвенный контроль электрической величины, пропорциональной амплитуде механических колебаний, обеспечивает возможность управления амплитудой колебаний рабочего инструмента для установления ее необходимого значения и поддержания этого значения в процессе длительного кавитационного воздействия при всех возможных изменениях параметров сред, условий обработки и изменений свойств и параметров инструментов. 


\section{Управление эффективностью} кавитационного воздействия

Вместе с тем возможность управления и стабилизации амплитуды механических колебаний рабочего инструмента не обеспечивают формирования в зазоре между излучателем и образцом с исследуемым материалом или покрытием одинакового по эффективности кавитационного воздействия. Обусловлено это тем, что кавитационная прочность различных жидких сред существенно различается и для формирования равного количества кавитационных пузырьков, одинаковых по запасаемой энергии в процессе формирования, тратиться различная энергия ультразвуковых колебаний [8]. Кроме того, размер области формирования кавитационых пузырьков вблизи излучающей поверхности зависит от вязкости обрабатываемой жидкой среды и от количества формируемых парогазовых пузырьков, ограничивающих выход упругих колебаний из излучателя в среду. Взрывающиеся кавитационные пузырьки формируют ударные волны и коммулятивные струи, которые должны достигать поверхности исследуемого образца, обладая максимальной разрушающей силой при достижении.

В связи с этим количество энергии, затрачиваемое на формирование максимально эффективного (по разрушающей силе) кавитационного облака, должно иметь оптимальное значение. Кроме того, необходимо учитывать влияние на эффективность кавитационного облака величины зазора между излучателем и разрушаемым материалом. Наличие оптимальных промежутков при формировании кавитационных процессов подтверждается многочисленными исследованиями.

То есть необходимо создавать определенную концентрацию парогазовых пузырьков, непрерывно возникающих и взрывающихся в зазоре между излучателем и разрушаемым образцом таким образом, чтобы формируемые при этом ударные волн и куммулятивные струи достигали исследуемого образца, осуществляя его максимальное разрушение.

Следовательно, ультразвуковой аппарат для исследования кавитационной прочности материалов и покрытий должен, во-первых, обеспечивать преобразование электрической энергии в акустическую, а вовторых, максимально эффективно осуществлять ее вывод в озвучиваемую акустическую нагрузку (среду).

Как было отмечено ранее, любая среда, в том числе жидкие среды (сплошные, разрыхленные кавитационными процессами и т. п.), рассматривается как своеобразная нагрузка на УЗКС. Все жидкие среды характеризуются плотностью, скоростью распространения акустической волны в среде, волновым сопротивлением озвучиваемой среды, которое определяется как произведение ее плотности на скорость распространения в ней УЗ волны, упругими свойствами озвучиваемой среды, вязкостью озвучиваемой среды и коэффициентом затухания в ней акустической волны.

Следует понимать, что все перечисленные свойства и характеристики жидкостей в УЗ полях не могут меняться, то есть любая акустическая среда, как аку- стическая нагрузка на УЗКС, является комплексной. Комплексный характер акустической нагрузки обусловлен трением частиц среды (активная составляющая). Реактивные свойства жидкостей (инерционные и упругие) определяют реактивную составляющую комплексной акустической нагрузки на УЗКС.

Существующая взаимосвязь между параметрами и характеристиками УЗКС и различными свойствами жидких сред, в том числе и кавитирующих в акустических полях, позволяет осуществлять непрерывный косвенный контроль свойств озвучиваемых сред.

Следует отметить, что акустическая нагрузка на УЗКС обусловлена не только свойствами озвучиваемых сред, но и геометрическими параметрами озвучиваемого объема.

Bсе это говорит о том, что наличие акустической нагрузки и изменение ее величины влияют на условия согласования УЗКС с озвучиваемой средой.

Определение значений элементов $R_{\mathrm{H}}, L_{\mathrm{H}}, C_{\mathrm{H}}$ с одной стороны позволит оперативно контролировать физические процессы, протекающие в озвучиваемой среде, а с другой стороны позволит оперативно выполнять подстройку параметров элементов согласующего контура (LC).

Ниже представлены выражения для вычисления значений $R_{\mathrm{H}}, L_{\mathrm{H}}, C_{\mathrm{H}}$ :

$R_{\mathrm{H}}=\frac{U_{\mathrm{H}}}{I_{\mathrm{H}}}-\frac{U_{0}}{I_{0}}, L_{\mathrm{H}}=\frac{Q_{\mathrm{H}} U_{\mathrm{H}}}{\omega_{\mathrm{H}} I_{\mathrm{H}}}-\frac{Q_{0} U_{0}}{\omega_{0} I_{0}}, C_{\mathrm{H}}=\left[\frac{\omega_{\mathrm{H}} Q_{\mathrm{H}} U_{\mathrm{H}}}{I_{\mathrm{H}}}-\frac{\omega_{0} Q_{0} U_{0}}{I_{0}}\right]^{-1}$,

где параметры $U_{0}$ и $I_{0}$ являются электрическим напряжением на пьезоэлементах УЗКС и током динамической ветви УЗКС (измеряются в режиме холостого хода на частоте $\left.\omega_{0}\right)$; параметры $U_{\text {н }}, I_{\text {н }}$ являются электрическим напряжением на пьезоэлементах УЗКС и током динамической ветви УЗКС (измеряются в режиме нагрузки на частоте $\left.\omega_{\text {н }}\right) ; Q_{0}$ - добротность УЗКС при отсутствии акустической нагрузки (собственная добротность УЗКС), $Q_{\text {н }}$ - добротность УЗКС, измеренная в режиме акустической нагрузки.

Добротность ненагруженной и нагруженной УЗКС $\left(Q_{0}, Q_{\mathrm{H}}\right)$ определяется по амплитудно-частотным характеристикам тока динамической ветви УЗКС ненагруженной и нагруженной УЗКС соответственно.

На рис. 3 проиллюстрирована разработанная структура системы контроля параметров озвучиваемых жидких сред.

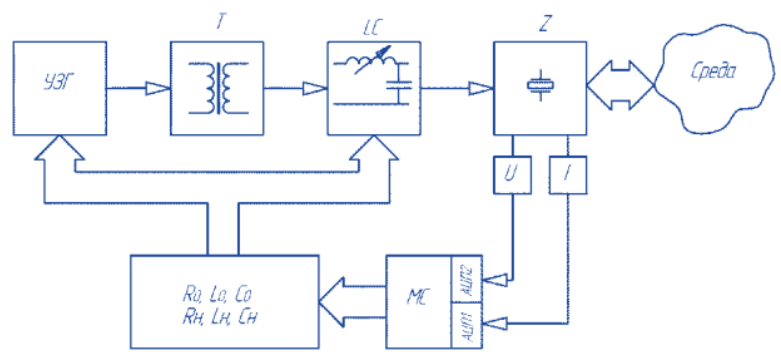

Pис. 3. Структурная схема измерительной части ультразвукового электронного генератора

Fig. 3. Block diagram of the measuring part of the ultrasonic electronic generator 
Измерительная схема состоит из узла выделения тока динамической ветви I и узла контроля питающего напряжения на УЗКС (U), сигналы с выхода которых поступают на управляющий микроконтроллер (MC).

Управляющий микроконтроллер, получая первичную измерительную информацию, управляет работой всех систем УЗ генератора, осуществляет вычисление параметров физической схемы замещения УЗКС, вырабатывает сигналы управления блоком согласования (LC).

Реализация управления для установления максимально эффективного кавитационного воздействия осуществляется следующим образом.

При предварительно установленном зазоре, рекомендованном стандартным методом (0...5 мм), осуществляется постепенное увеличение амплитуды УЗ воздействия с одновременным измерением значения сопротивления элемента $R H$ (эффективности кавитационного процесса) (рис. 4).

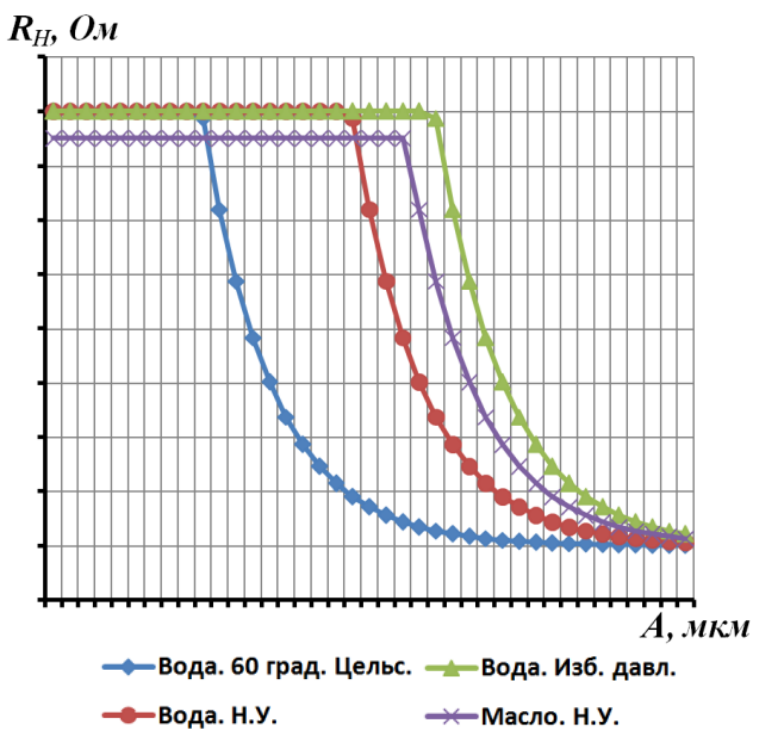

Рис. 4. Типичные зависимости активного сопротивления различных сред в различных условия от амплитудь УЗ воздействия

Fig. 4. Typical dependences of the active resistance of different media under different conditions on the amplitude of the ultrasonic impact

Характер изменения обусловлен развитием в жидких средах кавитационных процессов, насыщением жидкой среды парогазовыми пузырьками, начиная от стадии зарождающейся кавитации до стадии развитой кавитации.

Из представленных зависимостей следует, что при малых значениях амплитуды УЗ воздействия, значения сопротивлений элементов $R_{H}$ для различных сред и условий отличаются друг от друга. Однако по мере увеличения амплитуды УЗ воздействия (развития в жидкой среде кавитации) все кривые устремляются к одному уровню $R$, соответствующему режиму излучения в кавитирующую воду при нормальных условиях.
Кроме того, порог зарождения кавитации в различных жидких средах, а также для различных условий (температура, давление), различен. Изменяя величину зазора между исследуемым образцом, можно в некоторых пределах изменять величину акустического сопротивления среды, оптимизируя, таким образом, эффективность кавитационного воздействия.

На практике контроль кавитационных процессов в жидких средах может быть основан на количественной оценке крутизны $K$ кривых, представленных на рис. 4. На рис. 5 качественно показано изменение активного сопротивления $R_{H}$ от амплитуды УЗ воздействия $A$, где $R_{1}$ и $R_{2}$ - значения сопротивления механической ветви, обуславливаемые акустической нагрузкой, полученные для амплитуд УЗ воздействия $A_{1}$ и $A_{2}$ соответственно. Крутизна кривой $K$ для амплитуд в диапазоне $A_{1}, A_{2}$ определяется как:

$$
K=\frac{\left|R_{1}-R_{2}\right|}{\left|A_{1}-A_{2}\right|} \text {. }
$$

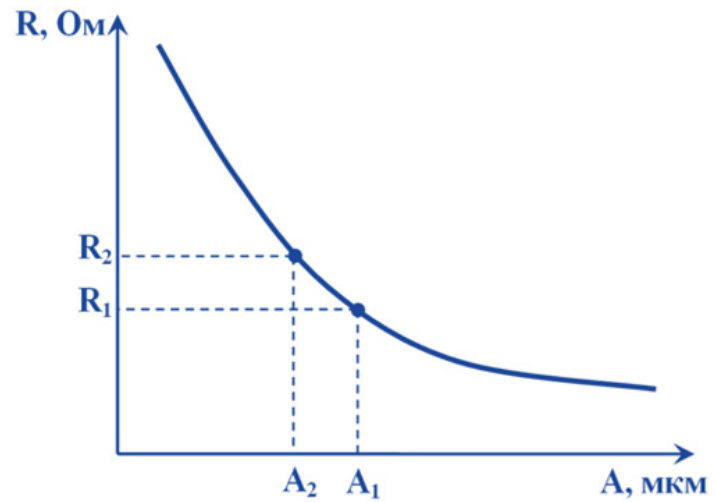

Рис. 5. Определение крутизны изменения активного сопротивления средь от амплитудьл УЗ воздействия

Fig. 5. Determination of the medium active resistance change steepness on the ultrasonic impact amplitude

Таким образом, задаваясь конкретным значением

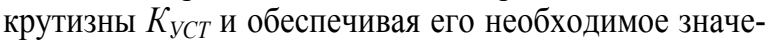
ние для различных жидких сред и внешних условий, можно обеспечить одинаковый уровень кавитационного воздействия на любую жидкую среду.

Предложенная и разработанная система контроля акустической нагрузки позволила обеспечить ее оперативный и косвенный контроль, а изменение величины зазора между излучающей поверхностью инструмента и образцом, а также изменение параметров электронного генератора (напряжения питания УЗ излучателя) позволили обеспечить одинаковый уровень кавитационного воздействия на различные материалы в различных средах и условиях.

\section{Заключение}

Для создания, исследования и применения специальных материалов и покрытий, способных обеспечить длительную надежную эксплуатацию оборудования предложен и разработан новый способ контроля эрозионной стойкости металлов и защитных 
покрытий при кавитационном воздействии в аномальных, по давлению и температуре, эксплуатационных условиях, расширяющий возможности стандарта ASTM G32-10 «Стандартный метод испытаний кавитационной эрозии».

Для реализации способа контроля предложен специализированный ультразвуковой аппарат, пригодный для проведения испытаний и выявления режимов кавитационного разрушения различных металлов и их покрытий при высоких давлениях, температуре до 1000 градусов Цельсия и в различных по свойствам жидких средах (в том числе, агрессивных).

Для создания необходимых условий ультразвукового воздействии (амплитуды колебаний и поддержания этого значения в процессе длительного кавитационного воздействия при всех возможных изменениях параметров сред) применена система управления амплитудой колебаний рабочего инструмента, основан-

\section{СПИСОК ЛИТЕРАТУРЫ}

1. Cavitation erosion resistance of $316 \mathrm{~L}$ austenitic steel processed by selective laser melting (SLM) / C. Hardesa, F. Pohl, A. Rottger, M. Thiele, W. Theisen, C. Esen // Wear. - 2019. - V. 426-427. URL: https://doi.org/10.1016/j.addma.2019.100786 (дата обращения 04.09.2020).

2. Romero M.C., Tschiptschin A.P., Scandian C. Cavitation erosion resistance of a non-standard cast cobalt alloy: influence of solubilizing and cold working treatments // Wear. - 2019. V. 426-427. URL: https://doi.org/10.1016/j.wear.2018.12.044 (дата обращения 04.09.2020)

3. Il-Cho P., Seong-Jong K. Effect of stabilizer concentration on the cavitation erosion resistance characteristics of the electroless nickel plated gray cast iron in seawater // Surface and Coatings Technology. - 2019. - V. 376. URL: https://doi.org/10.1016 j.surfcoat.2018.08.098 (дата обращения 04.09.2020).

4. Установка для испытания материалов на стойкость против кавитационной эрозии в криогенных средах: пат. Рос. Федерация № 351149 ; заявл. 09.02.1966; опубл. 29.02.1968. Бюл. № 27. -4 c.

5. Evaluation of cavitation erosion resistance of arc-sprayed Fe-based amorphous/nanocrystalline coatings in $\mathrm{NaCl}$ solution / Jinran Lina, Zehua Wang, Jiangbo Cheng, Min Kang, Xiuqing Fu, Sheng Hong // Results in Physics. - 2019. - V. 12. URL: https://doi.org/10.1016/ j.rinp.2018.12.007 (дата обращения 04.09.2020).

6. Improvement in cavitation erosion resistance of AA5083 aluminium alloy by laser shock processing / Zhaopeng Tong, Jiafei Jiao, Wangfan Zhou, Yu Yang Lan, Chen Huaile Liu, Yuzhou Sun, Xudong Ren // Surface and Coatings Technology. - 2019. V. 377. URL: https://doi.org/10.1016/j.surfcoat.2019.07.023 (дата обращения 04.09.2020).

7. Improvement of surface resistance to cavitation corrosion of nickel aluminum bronze by electropulsing-assisted ultrasonic surface rolling process / S. Yongan, W. Haibo, L. Wei, S. Guolin, Li Qiulin // Surface and Coatings Technology. - 2019. - V. 368. URL: https://doi.org/10.1016/j.surfcoat.2019.03.045 (дата обрашения 04.09.2020).

8. Lamana M.S., Pukasiewicz G.M.A., Sampath S. Influence of cobalt content and HVOF deposition process on the cavitation erosion resistance of WC-Co coatings // Wear. - 2019. - V. 398-399. URL: https://doi.org/10.1016/j.wear.2017.12.009 (дата обращения 04.09.2020).

9. Investigation of cavitation erosion resistance of AlSi10Mg alloy for additive manufacturing / L. Girelli, M. Tocci, L. Montesano, M. Gelfi, A. Pola // Wear. - 2019. - V. 402-403. URL: https://doi.org/10.1016/j.wear.2018.02.018 (дата обращения 04.09.2020). ная не непрерывном контроле электрических параметров пьезоэлектрической колебательной системы.

Для обеспечения равного по эффективности кавитационного процесса (степени его развития) в зазоре между излучающим инструментом и исследуемой поверхностью в различных средах и при различных условиях реализовано управление процессом, основанное на контроле изменения активного сопротивления среды при ее приближении к активному сопротивлению воды при нормальных условиях.

Практическая реализация испытаний кавитационной эрозии металлов и защитных покрытий в аномальных условиях обеспечит создание и применение новых материалов для использования в различных отраслях деятельности человека, промышленности.

Исследование выполнено при финансовой поддержке РФФИ и Госкорпорации «Росатом» в рамках научного проекта № 20-011-70001.

10. Muñoz-Cubillos J., Coronado J.J., Rodríguez S.A. On the cavitation resistance of deep rolled surfaces of austenitic stainless steels // Wear. - 2019. - V. 428-429. URL: https://doi.org/ 10.1016/j.wear.2019.03.001 (дата обращения 04.09.2020).

11. Resistance of curved surfaces to the cavitation erosion produced through high-pressure submerged waterjet / Mingming Zhou, Haixia Liu, Can Kang, Xiao Wei // Wear. - 2019. - V. 440-441. URL: https://doi.org/10.1016/j.wear.2019.203091 (дата обращения 04.09.2020).

12. Study of the cavitation resistance of Sn-based coatings / I. Tudela, R. Verbickas, G. Burmistroviene, Yi Zhang // Wear. - 2019. V. 410-411. URL: https://doi.org/10.1016/j.wear.2018.07.002 (дата обращения 04.09.2020).

13. The effect of boron on cavitation resistance of $\mathrm{FeCrMnSiB}$ austenitic stainless steels / J.R. Cruz, S.L. Henke, G.M.A. Pukasiewicz, A.N. D'Oliveira // Wear. - 2019. - V. 436-437. 2019. URL: https://doi.org/10.1016/j.wear.2019.203041 (дата обращения 04.09.2020).

14. Установка для испытания материалов на сопротивление кавитационной эрозии: пат. Рос. Федерация. №212591; заявл. 03.02.1966; опубл. 29.02.1968. - Бюл. № 9. - 2 с .

15. Устройство для исследования стойкости материалов против кавитационной эрозии: пат. Рос. Федерация. № 968696; заявл. 22.04.1981; опубл. 23.10.1982. - Бюл. № 39. - 3 с.

16. ASTM G32-10, Standard test method for cavitation erosion using vibratory apparatus ASTM International: West Conshohocken, PA. 2010 URL: www.astm.org (дата обращения 14.09.2020).

17. Ultrasonic device designed for the studying of cavitation resistance of materials / V.N. Khmelev, Yu.M. Kuzovnikov, S.S. Khmelev, S.V. Levin, M.V. Khmelev // Proc. 17th Int. Conf. of Young Specialists on Micro/Nanotechnologies and Electron Devices, EDM 2016. - Erlagol, Russian Federation: IEEE, 2016. - P. 260-263.

18. Устройство для исследования кавитационной прочности материалов: пат. Рос. Федерация № 163845, заявл: 08.02.2016, опубл. 10.08.2016, Бюл. № 22. -8 c.

19. Устройство для ультразвуковой обработки расплава легких сплавов: пат. Рос. Федерация №2719820, заявл.09.12.2019, опубл. 23.04.2020, Бюл. № 12. $-8 \mathrm{c}$.

20. Specific features of the realization of ultrasonic action in liquid media under excessive pressure / V.N. Khmelev, E.V. Ilchenko, R.V. Barsukov, D.V. Genne, S.F. Ryzhova // Proc. 20th Int. Conf. of Young Specialists on Micro/Nanotechnologies and Electron Devices, EDM 2019. - Erlagol, Russian Federation: IEEE, 2019. P. 235-239.

Поступила 09.09.2020 г. 


\section{Информация об авторах}

Хмелев В.Н., доктор технических наук, профессор кафедры методов и средств измерений и автоматизации, заместитель директора по науке Бийского технологического института Алтайского государственного технического университета им. И.И. Ползунова.

Барсуков P.B., кандидат технических наук, доцент кафедры методов и средств измерений и автоматизации Бийского технологического института Алтайского государственного технического университета им. И.И. Ползунова.

Голых $\boldsymbol{P . H . , ~ к а н д и д а т ~ т е х н и ч е с к и х ~ н а у к , ~ д о ц е н т ~ к а ф е д р ы ~ м е т о д о в ~ и ~ с р е д с т в ~ и з м е р е н и и ̆ ~ и ~ а в т о м а т и з а ц и и ~ Б и и ̆ - ~}$ ского технологического института Алтайского государственного технического университета им. И.И. Ползунова.

Абраменко Д.С., кандидат технических наук, доцент кафедры методов и средств измерений и автоматизации Бийского технологического института Алтайского государственного технического университета им. И.И. Ползунова.

Генне Д.В., инженер кафедры методов и средств измерений и автоматизации Бийского технологического института Алтайского государственного технического университета им. И.И. Ползунова.

Тертишников П.П., студент кафедры методов и средств измерений и автоматизации Бийского технологического института Алтайского государственного технического университета им. И.И. Ползунова. 
UDC 62-97/98

\title{
METHOD AND MEANS OF TESTING CAVITATION EROSION UNDER ABNORMAL CONDITIONS
}

\author{
Vladimir N. Khmelev ${ }^{1}$, \\ vnh@bti.secna.ru \\ Roman V. Barsukov 1 , \\ roman@bti.secna.ru \\ Roman N. Golykh', \\ grn@bti.secna.ru \\ Denis S. Abramenko ${ }^{1}$ \\ ades@bti.secna.ru \\ Dmitry V. Genne ${ }^{1}$, \\ gdv@bti.secna.ru
}

\author{
Pavel P. Tertishnikov ${ }^{1}$ \\ tertishnikov.pp@bti.secna.ru \\ 1 Polzunov Altai State Technical University, \\ 27, Trofimov street, Biysk, 659305, Russia.
}

The relevance of the research is caused by the need to study the resistance of metals and their coatings to cavitation erosion in conditions of abnormally high temperatures, pressures, as well as in chemically aggressive environments in the form of acids, alkalis or aggressive gases (such as ozone).

The main aim of the research is to develop specialized equipment for monitoring and detecting modes and dependencies of cavitation destruction of various metals and their coatings at abnormally high temperatures and overpressure, as well as methodology for determining (controlling) the resistance of metals and their coatings in cavitation fields.

Objects: testing cavitation erosion under abnormal conditions using specially designed ultrasonic equipment for this task.

Method: experimental method for studying cavitation erosion under abnormal conditions. This method is the development of the existing one regulated by the ASTM G32-10 «Standard test method for cavitation erosion».

Results. To create, research and apply special materials and coatings that can ensure long-term reliable equipment operation the authors have proposed and developed a new method for monitoring the erosion resistance of metals and protective coatings under cavitation exposure in abnormal operating conditions, in terms of pressure and temperature. This method expands the capabilities of the ASTM G32-10 «Standard test method for cavitation erosion». Practical implementation of tests of cavitation erosion of metals and protective coatings under abnormal conditions will ensure the creation and application of new materials for use in various branches of human activity and industry.

\section{Keywords:}

Ultrasonic impact, acoustic field, erosion, extreme conditions, frequency, normal conditions, amplitude.

The reported study was funded by RFBR and ROSATOM, project number 20-011-70001.

\section{REFERENCES}

1. Hardesa C., Pohl F., Rottger A., Thiele M., Theisen W., Esen C. Cavitation erosion resistance of $316 \mathrm{~L}$ austenitic steel processed by selective laser melting (SLM). Wear, 2019, vol. 426-427. Available at: https://doi.org/10.1016/j.addma.2019.100786 (accessed 4 September 2020).

2. Romero M.C., Tschiptschin A.P., Scandian C. Cavitation erosion resistance of a non-standard cast cobalt alloy: Influence of solubilizing and cold working treatments. Wear, 2019, vol. 426-427. Available at: https://doi.org/10.1016/j.wear.2018.12.044 (accessed 4 September 2020).

3. Il-Cho P., Seong-Jong K. Effect of stabilizer concentration on the cavitation erosion resistance characteristics of the electroless nickel plated gray cast iron in seawater. Surface and coatings technology, 2019, vol. 376. Available at: https://doi.org/10.1016/ j.surfcoat.2018.08.098 (accessed 4 September 2020).

4. Korobeynikov V.P. Ustanovka dlya ispytaniya materialov na stoykost protiv kavitatsionnoy erozii v kriogennykh sredakh [Installation for testing materials for resistance to cavitation erosion in cryogenic environments]. Patent RF, no. 351149, 1968.

5. Jinran Lina, Zehua Wang, Jiangbo Cheng, Min Kang, Xiuqing Fu, Sheng Hong. Evaluation of cavitation erosion resistance of arcsprayed Fe-based amorphous/nanocrystalline coatings in $\mathrm{NaCl}$ so- lution. Results in Physics, 2019, vol. 12. Available at: https:// doi.org/10.1016/j.rinp.2018.12.007 (accessed 4 September 2020).

6. Zhaopeng Tong, Jiafei Jiao, Wangfan Zhou, Yu Yang Lan, Chen Huaile Liu, Yuzhou Sun, Xudong Ren. Improvement in cavitation erosion resistance of AA5083 aluminium alloy by laser shock processing. Surface and Coatings Technology, 2019, vol. 377. Available at: https://doi.org/10.1016/j.surfcoat.2019.07.023 (accessed 4 September 2020).

7. Yongan S., Haibo W., Wei L., Guolin S. Qiulin Li. Improvement of surface resistance to cavitation corrosion of nickel aluminum bronze by electropulsing-assisted ultrasonic surface rolling process. Surface and coatings technology, 2019, vol. 368. Available at: https://doi.org/10.1016/j.surfcoat.2019.03.045 (accessed 4 September 2020)

8. Murilo S.L., Pukasiewicz G.M.A., Sanjay S. Influence of cobalt content and HVOF deposition process on the cavitation erosion resistance of WC-Co coatings. Wear, 2019, vol. 398-399. Available at: https://doi.org/10.1016/j.wear.2017.12.009 (accessed 4 September 2020)

9. Girelli L., Tocci M., Montesano L., Gelfi M., Pola A. Investigation of cavitation erosion resistance of AlSi10Mg alloy for additive manufacturing. Wear, 2019, vol. 402-403. Available at: https:// doi.org/10.1016/j.wear.2018.02.018 (accessed 4 September 2020). 
10. Muñoz-Cubillos J., Coronado J.J., Rodríguez S.A. On the cavitation resistance of deep rolled surfaces of austenitic stainless steels. Wear, 2019, vol. 428-429. Available at: https://doi.org/ 10.1016/j.wear.2019.03.001 (accessed 4 September 2020).

11. Mingming Zhou, Haixia Liu, Can Kang, XiaoWei. Resistance of curved surfaces to the cavitation erosion produced through highpressure submerged waterjet. Wear, 2019, vol. 440-441.Available at: https://doi.org/10.1016/j.wear.2019.203091 (accessed 4 September 2020).

12. Tudela I., Verbickas R., Burmistroviene G., Yi Zhang. Study of the cavitation resistance of Sn-based coatings. Wear, 2019, vol. 410-411. Available at: https://doi.org/10.1016/j.wear.2018.07.002 (accessed 4 September 2020).

13. Cruz J.R., Henke S.L., Pukasiewicz G.M.A., D’Oliveira A.N. The effect of boron on cavitation resistance of $\mathrm{FeCrMnSiB}$ austenitic stainless steels. Wear, 2019, vol. 436-437. Available at: https://doi.org/10.1016/j.wear.2019.203041 (accessed 4 September 2020).

14. Vardzyak P.F., Timerbulatov M.G. Ustanovka dlya ispytaniya materialov na soprotivlenie kavitatsionnoy erozii [Installation for testing materials for resistance to cavitation erosion]. Patent RF, no. $212591,1968$.

15. Konovalov V.M., Nikulin V.A., Rassomahin S.A. Ustroystvo dlya issledovaniya stoykosti materialov protiv kavitatsionnoy erozii [Device for studying the resistance of materials against cavitation erosion]. Patent RF, no. 968696, 1982.
16. ASTM G32-10, Standard Test Method for Cavitation Erosion Using Vibratory Apparatus ASTM International. West Conshohocken, PA. 2010 Available at: www.astm.org. (accessed 4 September 2020).

17. Khmelev V.N., Kuzovnikov Yu.M., Khmelev S.S., Levin S.V., Khmelev M.V. Ultrasonic device designed for the studying of cavitation resistance of materials.Proc. $17^{\text {th }}$ Int. Conf. of Young Specialists on Micro/Nanotechnologies and Electron Devices, EDM 2016. Erlagol, Russian Federation, IEEE, 2019. pp. 260-263.

18. Khmelev V.N., Kuzovnikov Yu.M., Tsyganok S.N., Khmelev M.V., Khmelev S.S., Shakura V.A. Ustroystvo dlya issledovaniya kavitatsionnoy prochnosti materialov [Device for research of cavitation]. Patent RF, no.163845, 2016.

19. Abramenko D.S., Genne D.V., Nesterov V.A., Tertishnikov P.P., Khmelev V.N., Khmelov M.V., Tsyganok S.N., Shalunov A.V. Ustroystvo dlya ultrazvukovoy obrabotki rasplava legkikh splavov [Device for ultrasonic treatment of light alloy melt]. Patent RF, no. $2719820,2020$.

20. Khmelev V.N., Ilchenko E.V., Barsukov R.V., Genne D.V., Ryzhova S.F. Specific features of the realization of ultrasonic action in liquid media under excessive pressure. Proc. $20^{\text {th }}$ Int. Conf. of Young Specialists on Micro/Nanotechnologies and Electron Devices, EDM 2019. Erlagol, Russian Federation, IEEE, 2019. pp. 235-239.

Received: 14 September 2020.

\section{Information about the authors}

Vladimir N. Khmelev, Dr. Sc., professor, deputy director for Science, Biysk Technological Institute of Polzunov Altai State Technical University.

Roman V. Barsukov, Cand. Sc., associate professor, Biysk Technological Institute of Polzunov Altai State Technical University.

Roman N. Golykh, Cand. Sc., associate professor, Biysk Technological Institute of Polzunov Altai State Technical University.

Denis S. Abramenko, Cand. Sc., associate professor, Biysk Technological Institute of Polzunov Altai State Technical University.

Dmitry V. Genne, engineer, Biysk Technological Institute of Polzunov Altai State Technical University.

Pavel P. Tertishnikov, student, Biysk Technological Institute of Polzunov Altai State Technical University. 